\title{
Thought-Action Fusion as Predictors of Obsessive-Compulsive Symptom Dimensions
}

\author{
Ji Eun Kim ${ }^{1,2}$ and Seung Jae Lee ${ }^{1,3 凶}$ \\ ${ }^{1}$ Department of Psychiatry, School of Medicine, Kyungpook National University, Daegu, Republic of Korea \\ ${ }^{2}$ Kim Ji Eun Psychiatric Clinic, Daegu, Republic of Korea \\ ${ }^{3}$ Department of Psychiatry, Kyungpook National University Hospital, Daegu, Republic of Korea
}

\begin{abstract}
Objective There have been several studies investigating the relationships between dysfunctional beliefs and obsessive-compulsive (OC) symptoms in obsessive-compulsive disorder (OCD). However, studies about the relationships between dysfunctional beliefs, especially thought-action fusion (TAF), and OC symptom dimensions have been scarce. Therefore, this study examined to what extent and how TAF subcomponents account for unique variability in four OC symptom dimensions.

Methods Sixty-five patients with OCD and 45 healthy controls aged between 18 and 30 years completed measures for OC symptom dimensions, OC symptoms, and dysfunctional beliefs such as TAF, trait-guilt, and inflated responsibility.

Results Three facets of TAF were exclusively associated with two symptom domains, namely, responsibility for harm and unacceptable thoughts, and explained the additional but small amount of variance to predict these two domains. In particular, the likelihood-others TAF positively predicted the unacceptable thoughts domain, whereas the likelihood-self TAF negatively predicted the aforementioned domain. For OC symptoms measured by the OC Inventory, no TAF components predicted the corresponding obsessing and mental neutralizing symptoms.

Conclusion This study provides supporting evidence that the three TAF subcomponents may be differently associated with certain OC symptom dimensions, and a dimensional approach may complement typical symptom-oriented OC measures.
\end{abstract}

Psychiatry Investig 2020;17(12):1226-1235

Key Words Cognitive theory, Dysfunctional belief, Cognitive appraisal, Obsessive-compulsive disorder, Metacognition.

\section{INTRODUCTION}

Obsessive-compulsive disorder (OCD) is a heterogeneous condition characterized by recurrent intrusive thoughts, urges, or impulses (obsessions) that provoke anxiety and repetitive behaviors or mental acts (compulsions) aimed at preventing or reducing anxiety or distress. ${ }^{1}$ In contemporary cognitive theories, the role of dysfunctional beliefs have been highlighted in the development and maintenance of various obsessivecompulsive (OC) symptomatology. ${ }^{2-4}$ According to these models, specific contents of the beliefs and appraisals give rise

Received: July 30, 2020 Revised: September 20, 2020

Accepted: October 11, 2020

$\triangle$ Correspondence: Seung Jae Lee, MD, PhD

Department of Psychiatry, School of Medicine, Kyungpook National University, 680 Gukchaebosang-ro, Jung-gu, Daegu 41944, Republic of Korea

Tel: +82-53-200-5752, Fax: +82-53-426-5361, E-mail: jayleemd@knu.ac.kr

(c) This is an Open Access article distributed under the terms of the Creative Commons Attribution Non-Commercial License (https://creativecommons.org/licenses/bync/4.0) which permits unrestricted non-commercial use, distribution, and reproduction in any medium, provided the original work is properly cited. to specific obsessions and compulsions.

However, the remarkable diversity of OCD symptomatology has limited success in relating OC symptoms to certain dysfunctional beliefs, as has been done with OC symptoms to other parameters such as biological markers, genetic factors, or treatment response. ${ }^{5}$ In fact, previous studies investigating the relationships between these dysfunctional beliefs and OC symptoms have resulted in inconsistent findings in clinical ${ }^{6-8}$ and nonclinical samples. ${ }^{9,10}$

To deal with OCD heterogeneity, a dimensional approach has been proposed. ${ }^{11,12}$ In over two decades of research in this field, the most consistently replicated OC symptom dimensions include 1) obsessions with contamination and compulsive washing and cleaning; 2 ) obsessions about responsibility for causing harm or making mistakes and compulsive checking; 3) obsessions about order and symmetry and compulsive ordering and arranging; and 4) repugnant obsessive thoughts about sex, religion, and violence, along with compulsive mental rituals and other covert neutralizing strategies. ${ }^{6}$ In terms of 
measures for symptom dimension, one of the most popularly used scales is the dimensional Yale-Brown Obsessive Compulsive Scale (DY-BOCS), ${ }^{13}$ a revision of the Y-BOCS. More recently, Abramowitz et al. ${ }^{6}$ developed a proper scale for OC symptom dimensions, the Dimensional Obsessive-Compulsive Scale (DOCS), which refined the limitations of the DYBOCS. In the Y-BOCS, for example, the division of OCD severity into obsessions and compulsions is inaccurate; items such as resistance to and control over OC symptoms are poor measures of OCD severity. ${ }^{14}$ In contrast, the DOCS assesses multiple empirically based parameters of severity (frequency, avoidance, distress, and functional interference) for each of the four OC symptom dimensions. ${ }^{14}$ Additionally, the DOCS is unique in that it affords an assessment of OC symptoms based on function rather than form. Therefore, the DOCS may be better suited than other symptom measures to investigating the relationships between OCD symptoms and dysfunctional beliefs.

An early study, ${ }^{15}$ using the DOCS, with 135 OCD patients showed that certain obsessive beliefs, measured by the 44item Obsessive Beliefs Questionnaire (OBQ-44) predicted certain OC symptom dimensions, measured by the DOCS, consistent with cognitive-behavioral conceptual models: contamination symptoms as well as symptoms related to being responsible for harm were predicted by responsibility/threat estimation beliefs, unacceptable thoughts were predicted by importance/control of thoughts (ICT) beliefs, and symmetry symptoms were predicted by perfectionism/certainty (PC) beliefs. A different study with mixed samples including OCD patients partially supported prior findings: unacceptable thoughts and symmetry symptom dimension were predicted by ICT and $\mathrm{PC}$, respectively. ${ }^{16} \mathrm{~A}$ recent study of 92 individuals with OCD and anxiety disorders almost replicated the results of the Wheaton et al. ${ }^{15}$ study, even after adding two constructs of cognitive fusion and experiential avoidance to the regression model. ${ }^{17}$

However, though it is widely used as a comprehensive measure of OC beliefs, instrument revision has narrowed the OBQ44's scope of assessment so that potentially important content is omitted. Specifically, the OBQ-44 contains thought-action fusion (TAF) items but it may cover only TAF moral content but not TAF likelihood content. ${ }^{18}$ In fact, one study found that TAF-L predicted OC symptoms beyond those predicted by the OBQ- $44 .^{18}$

TAF is the belief that thought and outcome or action are linked and that 1) thinking about something increases its likelihood of occurrence (likelihood TAF, TAF-L), or 2) that thoughts are morally equivalent to actions (moral TAF, TAFM). ${ }^{19,20}$ The former is further divided into likelihood-self (TAFLS) and likelihood-other (TAF-LO). According to Salkovskis' model, patients with OCD not only feel intensely responsible for their obsessional impulses to harm others or their unacceptable sexual or other images but also feel guilty over blaming themselves for these impulses or images. ${ }^{4,19}$ TAF, as a metacognition, may play a fundamental role in these catastrophic misinterpretations. ${ }^{20}$ That is, both types of TAF are closely connected with an inflated sense of responsibility and feelings of guilt, which may exacerbate obsessional complaints by increasing avoidance of guilt-provoking situations, lowering resistance to compulsions, and possibly by further increasing the salience of unwanted intrusive thoughts. ${ }^{21}$ However, to what extent and how TAF components explain the additional variance beyond inflated responsibility and feelings of guilt, especially in terms of OC symptom dimensions, are still unclear. Therefore, though previous studies between dysfunctional beliefs measured by the OBQ-44 and symptom dimensions exhibited general relationships, there is still a need for further research to focus on a certain dysfunctional belief and its associated components in a detailed relationship with OC symptom dimensions. To our knowledge, there has been no study published investigating the exact relationships between TAF and OC dimension in patients with OCD.

Therefore, this study aimed to investigate the relationship between three components of TAF, inflated responsibility, and guilt and individual symptom dimensions in patients with OCD. We hypothesized that 1) TAF would be most strongly associated with an unacceptable thoughts dimension because this domain was consistently presaged by ICT beliefs, ${ }^{17}$ 2) TAF components would explain the additional significant variance in certain OC symptom dimensions beyond inflated responsibility and feelings of guilt; ${ }^{8}$ and 3) TAF-L, among subcomponents of TAF, would better predict significant variance of an unacceptable thoughts dimension than TAF-M, based on previous observations. $^{8}$

\section{METHODS}

\section{Participants}

This study included 65 OCD patients and 45 healthy controls. The 65 OCD patients were aged between 18 and 30 years and were diagnosed with OCD through the OCD clinic at Kyungpook National University Hospital. A Structured Clinical Interview for DSM-5 Disorders, Clinical Version (SCID$5-\mathrm{CV}$ ) was carried out to determine the presence of OCD and other comorbidities. Subjects were excluded if they had a current comorbid psychiatric diagnosis, including major depressive disorder, alcohol use disorder, schizophrenia spectrum and other psychotic disorders and mental retardation, neurological disease, or a history of head injury or medical illness with documented cognitive sequelae. 
A local subway advertisement and an online bulletin board at Kyungpook National University were used to recruit 45 healthy volunteers aged between 18 and 30 years. Psychiatric interviews were conducted to exclude those with existing psychiatric pathology, psychotic symptoms, mental retardation, neurological disease, and history of head injury or medical illness.

Two experienced psychiatrists (S.J.L. and J.E.K.) administered psychiatric interviews on all participants who provided written informed consent according to the procedures approved by the Institutional Review Board of Kyungpook National University Hospital (2018-04-029).

\section{Psychological measures}

\section{The Dimensional Obsessive-Compulsive Scale (DOCS)}

The DOCS6 is a 20-item self-reported measure that assesses the severity of the four most consistently replicated OC symptom dimensions: 1) contamination, 2) responsibility for harm and mistakes, 3) unacceptable thoughts, and 4) symmetry and ordering. Each item is rated on a $0-4$ scale, yielding a total score that ranges from 0 to 80 as well as individual subscale scores ranging from 0 to 20 . The DOCS subscales have excellent reliability in clinical samples and the measure corresponds well with other measures of OC symptoms. We used a validated Korean version of the DOCS..$^{22}$ The reliability of the DOCS subscales in the Korean version were in the excellent range (Cronbach's $\alpha=0.91-0.95$ ).

\section{The Obsessive-Compulsive Inventory-Revised (OCI-R)}

The OCI- $\mathrm{R}^{23}$ is an 18 -item self-reported questionnaire that evaluates the degree of distress caused by OCD symptoms. Each of the items is rated from "not at all" (coded as 0 ) to "extremely" (coded as 4), and three items constitute a subtype of OCD symptoms. Extensively used in research with clinical and nonclinical samples, the OCI-R assesses six symptom domains: washing, checking, obsessing, neutralizing, ordering, and hoarding. Subscale scores range from 0 to 12 . We used a validated Korean version of the OCI-R. ${ }^{24}$

\section{The Thought-Action Fusion Scale (TAFS)}

The TAFS $^{20}$ is a 19-item self-reported measure that assesses the tendency to fuse thoughts to behaviors. Each item is rated on a 5-point scale ranging from 0 (disagree strongly) to 4 (agree strongly). These items are subdivided into TAF-M (12 items) and TAF-L (seven items); TAF-L is further divided into TAFLO (four items) and TAF-LS (three items). Higher TAF scores reflect a stronger tendency toward TAF-like cognitions. We used a translated Korean version of the TAFS. ${ }^{25}$
The Obsessive Belief Questionnaire, 44 Items (OBQ-44)

The Obsessive-Compulsive Cognitions Working Group developed the OBQ-44 to measure beliefs considered important in the development and maintenance of OCD. ${ }^{9}$ An exploratory factor analysis showed four factors: perfectionism and intolerance of uncertainty, importance and control of thoughts, responsibility, and overestimation of threat. Among these factors, we only used the responsibility factor score..$^{25}$

\section{The Guilt Inventory (GI)}

The $\mathrm{GI}^{25,26}$ is a 45 -item, self-reported inventory to assess three domains of guilt: state-guilt, defined as "present guilty feelings based on current or recent transgressions"; trait-guilt, defined as "a continuing sense of guilt beyond immediate circumstances"; and moral standards, defined as "subscription to a code of moral principles without reference either to specific behaviors or overly specific beliefs." Responses were rated on a 5-point Likert-type scale, ranging from 1 (strongly agree) to 5 (strongly disagree). This measure has demonstrated strong internal consistency. In this study, only the trait-guilt domain, measured by 17 items, was used. ${ }^{25}$

\section{The Beck Depression Inventory (BDI)}

The $\mathrm{BDI}^{27}$ is a self-report measure containing 21 items on a 4-point scale from 0 (symptom absent) to 3 (severe symptoms). Scoring is achieved by adding the highest ratings for all 21 items. We used a validated Korean version of the BDI. ${ }^{28}$

\section{Statistical analysis}

Our data analysis approach included the following steps: first, we computed descriptive statistics for all variables and used the Kolmogorov-Smirnov test to determine normality of all psychological data; second, we computed zero-order correlations to investigate associations between the DOCS subscales and other variables; third, to examine the contributions of TAF and related variables in predicting OC dimensions and symptoms, five independent variables, such as three subscales of TAFS, responsibility subscale of OBQ-44, and a trait factor of GI were entered; then, a series of hierarchical regression analyses were performed to predict symptom dimension scores for each DOCS subscale in addition to scores of individual symptoms of OCI: for each regression, two variables of OBQ-responsibility and trait-guilt as predictors were entered in the first step, and three subscales of TAFS were entered simultaneously in the second step. In addition, we performed a multicollinearity test on each independent variable for each regression. Generally, tolerance values less than 0.1 and variance inflation factor (VIF) values greater than 10 indicate reasons for concern because of collinearity among variables. 


\section{RESULTS}

\section{Clinical characteristics}

Table 1 displays demographic and clinical data for the OCD and healthy control groups. The mean $[\underline{ \pm}$ standard deviation (SD)] age of the OCD patients and normal controls was 22.9 \pm 3.5 and $22.6 \pm 2.0$ years, respectively. In terms of age and sex, there were no group differences. The duration of illness was $4.8 \pm 3.8$ years. Of the 65 patients, the OCD sample included 28 naïve or unmedicated and 37 medicated patients; among the medicated patients, most patients were taking selective serotonin reuptake inhibitors (SSRIs) (27 for escitalopram, five for paroxetine, two for fluoxetine, and one for sertraline).

The OCD patients, compared to the healthy control group, had significantly higher scores on the DOCS and OCI-R, all of which fell within the clinical range (Table 1). Patients also reported higher levels of depressive symptoms. In regards to dysfunctional beliefs related to OCD, patients showed higher total and subscale scores for TAF, inflated sense of responsibility, and trait-guilt. Over half of the psychological variables were normally distributed; two DOCS (washing and symmetry dimensions), four OCI subscales (ordering, obsessing, neutralizing, and hoarding), and subscales such as TAF-LO, TAFLS, and trait-guilt deviated from normality.

Table 1. Demographic and psychological data for OCD patients and normal controls

\begin{tabular}{|c|c|c|c|c|}
\hline & \multirow{2}{*}{$\begin{array}{c}\text { OCD }(\mathrm{N}=65) \\
\text { Mean } \pm \text { SD }\end{array}$} & \multirow{2}{*}{$\begin{array}{c}\mathrm{NC}(\mathrm{N}=45) \\
\text { Mean } \pm \mathrm{SD}\end{array}$} & \multicolumn{2}{|c|}{ Statistics } \\
\hline & & & $\chi^{2} / \mathrm{t}$ & $\mathrm{p}$ \\
\hline Male/female (N) & $52 / 13$ & $41 / 4$ & 2.5 & 0.113 \\
\hline Age (year) & $22.9 \pm 3.5$ & $22.6 \pm 2.0$ & 0.5 & 0.596 \\
\hline Duration of illness (year) & $4.8 \pm 4.1$ & - & - & \\
\hline Duration of pharmacotherapy (year) & $1.9 \pm 2.6$ & - & - & \\
\hline \multicolumn{5}{|l|}{ Symptom measures } \\
\hline \multicolumn{5}{|l|}{ O-C Inventory-Revised } \\
\hline Washing & $5.1 \pm 3.6$ & $1.3 \pm 1.3$ & 6.8 & $<0.0001$ \\
\hline Checking & $6.1 \pm 3.7$ & $1.9 \pm 1.5$ & 7.4 & $<0.0001$ \\
\hline Obsessing & $8.7 \pm 2.8$ & $2.6 \pm 2.4$ & 12.4 & $<0.0001$ \\
\hline Ordering & $5.1 \pm 3.7$ & $2.6 \pm 2.4$ & 3.0 & 0.003 \\
\hline Neutralizing & $5.0 \pm 4.1$ & $1.7 \pm 2.0$ & 5.0 & $<0.0001$ \\
\hline Hoarding & $4.3 \pm 3.3$ & $2.8 \pm 1.9$ & 2.8 & $<0.0001$ \\
\hline Total & $34.3 \pm 13.9$ & $13.6 \pm 7.3$ & 9.2 & $<0.0001$ \\
\hline \multicolumn{5}{|l|}{ Dimensional O-C Scale } \\
\hline Contamination/washing & $6.2 \pm 4.6$ & $3.2 \pm 1.9$ & 4.1 & $<0.0001$ \\
\hline Responsibility for harm & $8.6 \pm 5.6$ & $3.0 \pm 2.7$ & 6.2 & $<0.0001$ \\
\hline Unacceptable thoughts & $9.4 \pm 5.4$ & $3.1 \pm 2.9$ & 7.2 & $<0.0001$ \\
\hline Symmetry/ordering & $5.6 \pm 5.7$ & $1.9 \pm 2.3$ & 4.1 & $<0.0001$ \\
\hline Total & $29.8 \pm 12.6$ & $11.4 \pm 7.5$ & 8.8 & $<0.0001$ \\
\hline Beck Depression Inventory & $18.3 \pm 9.0$ & $4.6 \pm 4.1$ & 9.4 & $<0.0001$ \\
\hline \multicolumn{5}{|l|}{ Dysfunctional belief-related measures } \\
\hline \multicolumn{5}{|l|}{ Thought-Action Fusion Scale } \\
\hline Moral & $19.1 \pm 11.4$ & $14.2 \pm 8.2$ & 2.5 & 0.014 \\
\hline Likelihood, other & $4.8 \pm 4.1$ & $2.1 \pm 3.1$ & 3.8 & $<0.0001$ \\
\hline Likelihood, self & $5.0 \pm 3.3$ & $2.1 \pm 2.6$ & 4.9 & $<0.0001$ \\
\hline Total & $28.8 \pm 15.6$ & $18.3 \pm 11.9$ & 4.0 & $<0.0001$ \\
\hline \multicolumn{5}{|l|}{ Obsessional Belief Questionnaire } \\
\hline Responsibility/Threat & $67.2 \pm 20.3$ & $54.8 \pm 12.2$ & 3.6 & $<0.0005$ \\
\hline \multicolumn{5}{|l|}{ Guilt inventory } \\
\hline Trait & $69.6 \pm 10.3$ & $56.5 \pm 8.9$ & 7.1 & $<0.0001$ \\
\hline
\end{tabular}

OCD: obsessive-compulsive disorder, NC: normal controls, SD: standard deviation, O-C: obsessive-compulsive 


\section{Zero-order correlations}

Table 2 shows the zero-order correlations between four symptom dimensions and dysfunctional beliefs. As can be seen in the zero-order correlations, the two symptom dimensions of responsibility for harm and unacceptable thoughts were associated with most subscale scores of TAF, responsibility, and guilt $(0.26 \leq \mathrm{r} \leq 0.50$ for responsibility for harm, $0.25 \leq$ $\mathrm{r} \leq 0.43$ for unacceptable thoughts domain). In particular, both symptom dimensions exhibited the highest correlation coefficients with responsibility belief. On the other hand, the other two dimensions of contamination/washing and symmetry/ ordering did not exhibit any relationship, except with depression scores.

Table 3 shows the zero-order correlations between six symptoms and dysfunctional beliefs. TAF-M was associated with obsessing symptom ( $\mathrm{r}=0.34, \mathrm{p}=0.005)$; TAF-LO was associated with mental neutralizing $(\mathrm{r}=0.34, \mathrm{p}=0.006)$; and TAF-LS was associated with checking $(r=0.25, p=0.048)$ and mental neutralizing symptoms $(\mathrm{r}=0.33, \mathrm{p}=0.008)$. The other three symptoms did not show any relationship with the three TAF subcomponents.

\section{Hierarchical multiple regression analysis predicting individual DOCS symptom dimensions}

Table 4 presents results of the regression analyses predicting each DOCS subscale.

In the first step of the regression analysis predicting DOCS contamination scores, two variables, namely, inflated responsibility and trait-guilt, explained the very small and nonsignificant amount of variance $\left(R^{2}=0.05, p=0.240\right)$. Inclusion of the

Table 2. Zero-order correlations between symptom dimensions and dysfunctional beliefs in OCD patients $(\mathrm{N}=65)$

\begin{tabular}{|c|c|c|c|c|c|c|c|c|c|}
\hline & 1 & 2 & 3 & 4 & 5 & 6 & 7 & 8 & 9 \\
\hline \multicolumn{10}{|l|}{ 1. DOCS-C } \\
\hline 2. DOCS-R & 0.08 & & & & & & & & \\
\hline 3. DOCS-U & -0.13 & $0.49^{\ddagger}$ & & & & & & & \\
\hline 4. DOCS-S & $0.26^{*}$ & -0.22 & 0.10 & & & & & & \\
\hline 5. TAF-M & -0.01 & $0.38^{\dagger}$ & $0.32^{\dagger}$ & 0.07 & & & & & \\
\hline 6. TAF-LO & 0.00 & $0.26^{*}$ & $0.25^{*}$ & -0.08 & 0.37 & & & & \\
\hline 7. TAF-LS & -0.06 & $0.34^{\dagger}$ & 0.08 & -0.11 & $0.43^{\ddagger}$ & $0.74^{\ddagger}$ & & & \\
\hline 8. OBQ-RT & -0.17 & $0.50^{\ddagger}$ & $0.43^{\ddagger}$ & 0.09 & 0.52 & $0.26^{*}$ & 0.25 & & \\
\hline 9. G-T & 0.02 & $0.29^{*}$ & $0.30^{*}$ & 0.19 & 0.14 & 0.07 & 0.12 & $0.47^{\ddagger}$ & \\
\hline 10. BDI & 0.12 & $0.48^{\ddagger}$ & $0.44^{\ddagger}$ & $0.36^{\dagger}$ & $0.30^{\dagger}$ & $0.38^{\dagger}$ & $0.39^{\dagger}$ & $0.43^{\dagger}$ & $0.68^{\ddagger}$ \\
\hline
\end{tabular}

${ }^{*} \mathrm{p}<0.05,{ }^{\dagger} \mathrm{p}<0.01,{ }^{\ddagger} \mathrm{p}<0.001$. DOCS-C, -R, -U, -S: Dimensional Obsessive-Compulsive Scale-Contamination, -Responsibility for harm, -Unacceptable thoughts, -Symmetry, TAF-M, -LO, -LS: Thought-Action Fusion Scale-Moral, -Likelihood Others, -Likelihood Self, OBQ-RT: Obsessive Belief Questionnaire-Responsibility/Threat, G-T: Guilt inventory-Trait, BDI: Beck Depression Inventory

Table 3. Zero-order correlations between obsessive-compulsive symptoms and dysfunctional beliefs in OCD patients ( $\mathrm{N}=65)$

\begin{tabular}{|c|c|c|c|c|c|c|c|c|c|c|c|}
\hline & 1 & 2 & 3 & 4 & 5 & 6 & 7 & 8 & 9 & 10 & 11 \\
\hline \multicolumn{12}{|l|}{ 1. OCI-C } \\
\hline 2. OCI-H & $0.45^{\ddagger}$ & & & & & & & & & & \\
\hline 3. OCI-N & $0.48^{\ddagger}$ & $0.33^{\dagger}$ & & & & & & & & & \\
\hline 4. OCI-Ob & 0.15 & 0.14 & $0.37^{\dagger}$ & & & & & & & & \\
\hline 5. OCI-Or & $0.44^{\ddagger}$ & $0.50^{\ddagger}$ & $0.33^{\dagger}$ & 0.16 & & & & & & & \\
\hline 6. OCI-W & $0.26^{*}$ & 0.21 & $0.27^{\dagger}$ & 0.13 & $0.35^{\dagger}$ & & & & & & \\
\hline 7. TAF-M & 0.16 & 0.08 & 0.13 & $0.34^{\dagger}$ & 0.12 & 0.12 & & & & & \\
\hline 8. TAF-LO & 0.07 & 0.11 & $0.34^{\dagger}$ & 0.23 & 0.08 & 0.05 & $0.37^{\dagger}$ & & & & \\
\hline 9. TAF-LS & $0.25^{*}$ & 0.23 & $0.33^{\dagger}$ & 0.21 & 0.12 & -0.01 & $0.43^{\ddagger}$ & $0.74^{\ddagger}$ & & & \\
\hline 10. OBQ-RT & $0.28^{*}$ & 0.18 & $0.32^{\dagger}$ & $0.30^{*}$ & $0.30^{*}$ & 0.02 & $0.52^{\ddagger}$ & $0.26^{*}$ & $0.25^{*}$ & & \\
\hline 11. G-T & $0.25^{*}$ & $0.26^{*}$ & 0.22 & $0.37^{\dagger}$ & $0.39^{\dagger}$ & 0.04 & 0.14 & 0.07 & 0.12 & $0.47^{\ddagger}$ & \\
\hline 12. BDI & $0.27^{*}$ & 0.15 & $0.39^{\dagger}$ & $0.47^{\ddagger}$ & $0.34^{\dagger}$ & 0.09 & $0.26^{*}$ & 0.23 & 0.18 & $0.37^{\dagger}$ & $0.57^{\ddagger}$ \\
\hline
\end{tabular}

${ }^{*} \mathrm{p}<0.05,{ }^{\dagger} \mathrm{p}<0.01,{ }^{\ddagger} \mathrm{p}<0.001$. OCI-C, -H, -N, -Ob, -Or, W: Obsessive-Compulsive Inventory-Contamination, -Hoarding, -Neutralizing, -Obsessing, -Ordering, -Washing, TAFS-M, -LO, -LS: Thought-Action Fusion Scale-Moral, -Likelihood Others, -Likelihood Self, OBQ-RT: Obsessive Belief Questionnaire-Responsibility/Threat, G-T: Guilt inventory-Trait, BDI: Beck Depression Inventory 
Table 4. Multiple regression analysis (enter method) for predicting individual obsessive-compulsive symptom dimensions

\begin{tabular}{|c|c|c|c|c|c|c|c|c|c|c|c|c|}
\hline \multirow{2}{*}{ Variable } & \multicolumn{3}{|c|}{ Contamination $^{\mathrm{a}}$} & \multicolumn{3}{|c|}{ Responsibility for harm ${ }^{\mathrm{b}}$} & \multicolumn{3}{|c|}{ Unacceptable thoughts $^{c}$} & \multicolumn{3}{|c|}{ Symmetry $^{\mathrm{d}}$} \\
\hline & B & SE & $\beta$ & B & SE & $\beta$ & B & SE & $\beta$ & B & SE & $\beta$ \\
\hline \multicolumn{13}{|l|}{ Model 1} \\
\hline Constant & 5.47 & 3.86 & & -2.57 & 4.21 & & -1.79 & 4.17 & & -1.71 & 4.83 & \\
\hline OBQ-RT & -0.05 & 0.03 & -0.24 & 0.13 & 0.03 & $0.46^{\dagger}$ & 0.10 & 0.03 & $0.37^{\dagger}$ & 0.00 & 0.04 & 0.00 \\
\hline G-T & 0.06 & 0.06 & 0.14 & 0.04 & 0.07 & 0.07 & 0.07 & 0.07 & 0.13 & 0.10 & 0.08 & 0.19 \\
\hline \multicolumn{13}{|l|}{ Model 2} \\
\hline Constant & 5.08 & 3.95 & & -3.61 & 4.20 & & -2.53 & 4.07 & & -1.46 & 4.92 & \\
\hline OBQ-RT & -0.07 & 0.04 & -0.33 & 0.10 & 0.04 & $0.37^{*}$ & 0.06 & 0.04 & 0.24 & -0.01 & 0.05 & -0.03 \\
\hline G-T & 0.08 & 0.06 & 0.17 & 0.04 & 0.07 & 0.08 & 0.09 & 0.07 & 0.18 & 0.11 & 0.08 & 0.21 \\
\hline TAF-M & 0.06 & 0.06 & 0.16 & 0.05 & 0.07 & 0.09 & 0.09 & 0.07 & 0.19 & 0.06 & 0.08 & 0.13 \\
\hline TAF-LO & 0.15 & 0.21 & 0.13 & -0.04 & 0.22 & -0.03 & 0.49 & 0.22 & $0.37^{*}$ & -0.01 & 0.26 & -0.01 \\
\hline TAF-LS & -0.23 & 0.27 & -0.16 & 0.37 & 0.29 & 0.22 & -0.58 & 0.28 & $-0.36^{*}$ & -0.30 & 0.34 & -0.17 \\
\hline
\end{tabular}

${ }^{*} \mathrm{p}<0.05,{ }^{\dagger} \mathrm{p}<0.01 .{ }^{\mathrm{a}} \mathrm{R}^{2}=0.05, \mathrm{p}=0.24$ for Model $1, \Delta \mathrm{R}^{2}=0.02, \mathrm{p}=0.50$ for Model $2,{ }^{\mathrm{b}} \mathrm{R}^{2}=0.25, \mathrm{p}<0.001$ for Model $1, \Delta \mathrm{R}^{2}=0.05, \mathrm{p}=0.001$ for Model $2,{ }^{c} \mathrm{R}^{2}=0.19, \mathrm{p}=0.001$ for Model $1, \Delta \mathrm{R}^{2}=0.09, \mathrm{p}=0.001$ for Model $2,{ }^{\mathrm{d}} \mathrm{R}^{2}=0.04, \mathrm{p}=0.32$ for Model $1, \Delta \mathrm{R}^{2}=0.03$, $\mathrm{p}=0.54$ for Model 2. OBQ-RT: Obsessive Belief Questionnaire-Responsibility/Threat, G-T: Guilt inventory-Trait, TAF-M, -LO, -LS: Thought-Action Fusion Scale-Moral, -Likelihood Others, -Likelihood Self

three TAF subcomponents in step 2 also explained little additional variance $\left(\mathrm{R}^{2}\right.$ change $\left.=0.02, \mathrm{p}=0.500\right)$. In this model, no variables emerged as significant individual predictors of the DOCS contamination subscale scores.

In regards to the DOCS responsibility for harm dimension, the first step showed that the two variables accounted for a significant portion of the variance in this DOCS subscale $\left(\mathrm{R}^{2}=\right.$ $0.25, \mathrm{p}<0.001)$. In the first step, the only significant predictor of the DOCS responsibility for harm dimension was the dysfunctional belief of responsibility. In step 2, adding the three TAF variables accounted for a very small additional variance $\left(\mathrm{R}^{2}\right.$ change $\left.=0.05, \mathrm{p}=0.001\right)$. In this model, responsibility belief remained a significant predictor.

In regards to the DOCS unacceptable thoughts dimension, the step 1 model accounted for $19 \%$ of the variance in the DOCS unacceptable thoughts scores, which was strongly significant $\left(\mathrm{R}^{2}=0.19, \mathrm{p}=0.001\right)$. Inclusion of the three TAF variables in the second step explained the small but significant additional variance $\left(\mathrm{R}^{2}\right.$ change $\left.=0.09, \mathrm{p}=0.001\right)$. In the second step, TAF-LO and TAF-LS significantly predicted the DOCS unacceptable thoughts dimension but their directions were different; TAF-LO positively predicted the domain while TAFLS negatively did $(\beta=0.37$ and $p=0.028$ for TAF-LO; $\beta=-0.36$ and $\mathrm{p}=0.041$ for TAF-LS).

In regards to the DOCS symmetry dimension, the two variables explained a very small and nonsignificant amount of variance $\left(\mathrm{R}^{2}=0.05, \mathrm{p}=0.32\right)$. Inclusion of the three TAF variables in step 2 also explained nonsignificant additional variance $\left(\mathrm{R}^{2}\right.$ change $\left.=0.03, \mathrm{p}=0.54\right)$.

Regarding collinearity across regression analyses, tolerance values ranged from 0.36 to 0.77 and VIF values ranged from
1.29 to 2.76 . These results indicate that multicollinearity was not observed for all independent variables for each regression.

\section{Hierarchical multiple regression analysis predicting individual OCI symptoms}

Table 5 presents results of the regression analyses predicting each OCI symptom subscale.

In regards to the washing symptom, the two variables explained little amount of variance $\left(\mathrm{R}^{2}=0.001, \mathrm{p}=0.960\right)$. Inclusion of the three TAF variables in step 2 also explained a nonsignificant, very small additional variance $\left(R^{2}\right.$ change $=0.03$, $\mathrm{p}=0.889$ ) with no significant predictors.

In regards to the checking/doubting symptom, the two variables explained a small and significant amount of variance $\left(\mathrm{R}^{2}=\right.$ $0.10, \mathrm{p}=0.044$ ). Inclusion of the three TAF variables in step 2 also explained a nonsignificant, small additional variance $\left(\mathrm{R}^{2}\right.$ change $=0.06, \mathrm{p}=0.056$ ). TAF-LS emerged as one single significant predictor in the second step. Note that TAF-LO, though nonsignificant, exhibited negative prediction tendency.

In regards to the obsessing symptom, the step 1 model accounted for $16 \%$ of the variance in obsessing symptom scores $\left(\mathrm{R}^{2}=0.16, \mathrm{p}=0.005\right)$. Inclusion of the three TAF variables in step 2 also explained a small and significant additional variance $\left(\mathrm{R}^{2}\right.$ change $=0.07, \mathrm{p}=0.007)$. Trait-guilt emerged as a single significant predictor in the first and second steps.

In regards to the ordering score of the OCI-R, the two variables explained a small and significant amount of variance $\left(\mathrm{R}^{2}=0.16, \mathrm{p}=0.004\right)$. Inclusion of the three TAF variables in step 2 also explained a significant, very small additional variance $\left(\mathrm{R}^{2}\right.$ change $\left.=0.01, \mathrm{p}=0.047\right)$. Trait-guilt emerged as a single significant predictor in both steps. 
Table 5. Multiple regression analysis (enter method) for predicting individual obsessive-compulsive symptoms

\begin{tabular}{|c|c|c|c|c|c|c|c|c|c|c|c|c|c|c|c|c|c|c|}
\hline \multirow[t]{2}{*}{ Variable } & \multicolumn{3}{|c|}{ Washing ${ }^{\mathrm{a}}$} & \multicolumn{3}{|c|}{$\begin{array}{l}\text { Checking/ } \\
\text { doubting }^{\mathrm{b}}\end{array}$} & \multicolumn{3}{|c|}{ Obsessing $^{c}$} & \multicolumn{3}{|c|}{ Ordering $^{\mathrm{d}}$} & \multicolumn{3}{|c|}{$\begin{array}{c}\text { Mental } \\
\text { neutralizing }\end{array}$} & \multicolumn{3}{|c|}{ Hoarding $^{\mathrm{f}}$} \\
\hline & B & SE & $\beta$ & B & SE & $\beta$ & B & SE & $\beta$ & B & SE & $\beta$ & B & SE & $\beta$ & B & SE & $\beta$ \\
\hline \multicolumn{19}{|l|}{ Model 1} \\
\hline Constant & 4.25 & 3.09 & & -0.18 & 3.02 & & 1.70 & 2.19 & & -4.56 & 2.91 & & -1.09 & 3.31 & & -1.57 & 2.78 & \\
\hline OBQ-RT & 0.00 & 0.03 & 0.01 & 0.04 & 0.02 & 0.20 & 0.02 & 0.02 & 0.16 & 0.03 & 0.02 & 0.15 & 0.06 & 0.03 & $0.28^{*}$ & 0.01 & 0.02 & 0.07 \\
\hline G-T & 0.01 & 0.05 & 0.03 & 0.06 & 0.05 & 0.16 & 0.08 & 0.04 & $0.30^{*}$ & 0.11 & 0.05 & $0.32 *$ & 0.03 & 0.05 & 0.08 & 0.07 & 0.04 & 0.23 \\
\hline \multicolumn{19}{|l|}{ Model 2} \\
\hline Constant & 3.93 & 3.15 & & -0.40 & 3.00 & & 1.04 & 2.16 & & -4.62 & 3.00 & & -1.66 & 3.23 & & -1.83 & 2.80 & \\
\hline OBQ-RT & -0.02 & 0.03 & -0.09 & 0.04 & 0.03 & 0.20 & 0.00 & 0.02 & -0.03 & 0.03 & 0.03 & 0.15 & 0.06 & 0.03 & 0.28 & 0.01 & 0.03 & 0.07 \\
\hline G-T & 0.02 & 0.05 & 0.06 & 0.05 & 0.05 & 0.13 & 0.09 & 0.04 & $0.34^{*}$ & 0.11 & 0.05 & $0.31^{*}$ & 0.03 & 0.05 & 0.07 & 0.07 & 0.05 & 0.21 \\
\hline TAF-M & 0.06 & 0.05 & 0.18 & -0.01 & 0.05 & -0.03 & 0.06 & 0.04 & 0.26 & -0.01 & 0.05 & -0.03 & -0.06 & 0.05 & -0.18 & -0.02 & 0.05 & -0.08 \\
\hline TAF-LO & 0.10 & 0.17 & 0.11 & -0.24 & 0.16 & -0.27 & 0.10 & 0.11 & 0.15 & -0.02 & 0.16 & -0.02 & 0.18 & 0.17 & 0.18 & -0.10 & 0.15 & -0.12 \\
\hline TAF-LS & -0.16 & 0.22 & -0.15 & 0.44 & 0.21 & $0.40^{*}$ & -0.04 & 0.15 & -0.05 & 0.08 & 0.21 & 0.07 & 0.23 & 0.22 & 0.19 & 0.31 & 0.19 & 0.31 \\
\hline
\end{tabular}

${ }^{*} \mathrm{p}<0.05$. ${ }^{\mathrm{a}} \mathrm{R}^{2}=0.001, \mathrm{p}=0.96$ for Model $1, \Delta \mathrm{R}^{2}=0.03, \mathrm{p}=0.90$ for Model $2,{ }^{b} \mathrm{R}^{2}=0.10, \mathrm{p}=0.04$ for Model $1, \Delta \mathrm{R}^{2}=0.06$, $\mathrm{p}=0.06$ for Model 2 , ${ }^{\mathrm{c}} \mathrm{R}^{2}=$ $0.16, p=0.01$ for Model $1, \Delta \mathrm{R}^{2}=0.07, \mathrm{p}=0.01$ for Model $2,{ }^{\mathrm{d}} \mathrm{R}^{2}=0.16, \mathrm{p}=0.01$ for Model $1, \Delta \mathrm{R}^{2}=0.01, \mathrm{p}=0.04$ for Model $2,{ }^{\mathrm{e}} \mathrm{R}^{2}=0.11, \mathrm{p}=0.03$ for Model 1, $\Delta \mathrm{R}^{2}=0.09, \mathrm{p}=0.02$ for Model $2,{ }^{\mathrm{f}} \mathrm{R}^{2}=0.07, \mathrm{p}=0.10$ for Model $1, \Delta \mathrm{R}^{2}=0.05, \mathrm{p}=0.18$ for Model 2. OBQ-RT: Obsessive Belief Questionnaire-Responsibility/Threat; G-T: Guilt inventory-Trait; TAF-M, -LO, -LS: Thought-Action Fusion Scale-Moral, -Likelihood Others, -Likelihood Self

In regards to mental neutralizing, the two variables of dysfunctional beliefs explained a significant amount of variance $\left(\mathrm{R}^{2}=0.11, \mathrm{p}=0.028\right)$, with inflated responsibility as a significant predictor. In step 2, addition of the three TAF variables increased the small variance accounted for neutralizing score ( $\mathrm{R}^{2}$ change $\left.=0.10, \mathrm{p}=0.015\right)$ without a significant predictor.

Regarding hoarding, the two variables explained a small and nonsignificant amount of variance $\left(\mathrm{R}^{2}=0.07, \mathrm{p}=0.101\right)$. Inclusion of the three TAF variables in step 2 also explained a nonsignificant, very small additional variance $\left(\mathrm{R}^{2}\right.$ change $=0.05$, $\mathrm{p}=0.180$ ). There was no significant prediction in both steps.

Regarding collinearity across regression analyses, tolerance values ranged from 0.42 to 0.75 and VIF values ranged from 1.33 to 2.40 . These results indicate that multicollinearity was not observed for all independent variables.

\section{DISCUSSION}

In the current study, we found that three facets of TAF were exclusively associated with the DOCS responsibility for harm and unacceptable thoughts dimension in young adults with OCD. Multiple regression analyses demonstrated that inclusion of the three new predictors of TAF subscales accounted for an additional but small amount of variance in these two dimensions. Specifically, TAF-LO positively predicted the unacceptable thoughts dimension, whereas TAF-LS negatively predicted the aforementioned dimension. However, any facet of TAF was not found as a significant predictor in other symptom dimensions. In contrast, as measured by the OCI, no TAF components predicted the obsessing and mental neutralizing symptoms, which are corresponding symptoms of the DOCS unacceptable thoughts dimension. Rather, TAF-LS was found to be the only significant predictor in the checking symptom.

\section{Relationships between TAF and OC symptom dimension}

In this study, three subcomponents of TAF, inflated responsibility, and trait-guilt correlated only with the DOCS responsibility for harm and unacceptable thoughts dimensions: none of these variables were associated with the DOCS washing and symmetry dimensions. These findings suggest that the five midstructures included in this study may relate more to the former two dimensions. However, regression analyses showed different patterns of predictors, though five variables accounted for about $30 \%$ of variance in both dimensions.

First, TAF-LO and TAF-LS were significant predictors only for the unacceptable thoughts dimension. To our knowledge, there has been no previous study on the direct relationship between the TAFS and DOCS in OCD patients. However, our findings were corroborated by two previous studies, one with $135 \mathrm{OCD}$ patients ${ }^{15}$ and the other with a mixed sample including 30 OCD patients, ${ }^{16}$ demonstrating that the OBQ-ICT subscale including some TAF items was the only significant predictor for an unacceptable thoughts dimension and did not predict any other dimensions. A recent study with 92 OCD patients also revealed the OBQ-ICT as a predictor for the unacceptable thoughts and contamination dimensions. ${ }^{17}$

In regards to TAF subcomponents, TAF-LO positively pre- 
dicted the unacceptable thoughts domain in this study. The TAF-LO and TAF-LS subcomponents have shown a more consistent significant correlation to OC symptoms, ${ }^{29}$ particularly TAF-LO ${ }^{20}$ Interestingly, however, TAF-LS predicted this domain in a negative direction (i.e., patients who reported more TAF-LS tended to score lower on the unacceptable thoughts domain). Additionally, in this study, the zero-order correlation between TAF-LS and this domain was near zero and nonsignificant. The reason for the negative association in the present clinical sample is unclear, though it could be argued that the unacceptable thoughts dimension is one greatly oriented toward others. Speculatively, this difference between increased and decreased TAF bias toward others and self, respectively, may exaggerate the preoccupation of others-oriented obsessive thoughts in patients with OCD. It should also be noted that, in spite of its negative prediction, TAF-LS was still higher in patients with OCD than in normal controls in this study. However, previous studies have shown that TAF-LS is generally non-specific to OCD compared to other clinical group ${ }^{30}$ and is relevant to symptoms reflecting multiple forms of psychopathology ${ }^{18}$ More studies with a large sample size are needed. In addition, we observed that TAF-M was not a significant predictor of this domain. Though subjects were nonclinical undergraduates, TAF-M did not predict the unacceptable thoughts domain while TAF-L did. ${ }^{31}$ The TAF moral facet generally does not show a specific relation to OC symptoms ${ }^{32}$ and, in fact, shows strong correlations with depression. ${ }^{30}$

In this study, we found that an inflated sense of responsibility was the only predictor for the DOCS responsibility for harm dimension. In this study, these two variables also showed the highest correlation coefficient $(\mathrm{r}=0.50)$ in correlation analysis. These findings are consistent with previous studies that show OBQ-RT was the most significant variable predicting the DOCS responsibility for harm dimension. ${ }^{15-17}$

\section{Relationships between TAF and OC symptoms}

We performed the same regression analyses, except the dependent symptom variables measured by the OCI, to ascertain the uniqueness of the dimensional approach. In general, compared to results from the DOCS, five variables accounted for a smaller amount of variance in individual symptoms, such as obsessing, ordering, and mental neutralizing, of which the models were significant in this study.

Regarding TAF, TAF-LS significantly predicted the checking/doubting symptom in a positive direction while TAF-LO tended to negatively predict this symptom, though nonsignificantly. In this study, these findings were reversed findings of TAF-LS and TAF-LO predicting the unacceptable thoughts dimension. Therefore, contrary to the unacceptable thoughts dimension, the checking/doubting symptom may be related to a self-focused TAF tendency. However, no TAF components predicted the obsessing and mental neutralizing symptoms, which are corresponding symptoms of the DOCS unacceptable thoughts dimension, though the OBQ-ICT has predicted the obsessing symptom across different investigations. ${ }^{7,8,33}$ Speculatively, the negative finding using the OCI may stem from the possibility that symptoms within the unacceptable thoughts dimension can be measured separately into two categories - mental neutralizing and obsessing symptoms-in the OCI. Unlike regression analyses, correlation analyses in this study showed that the three TAF subcomponents correlated almost exclusively and moderately with mental neutralizing and obsessing symptoms.

This study, instead of TAF, found trait-guilt as a significant predictor for both the obsessing and ordering symptoms. In terms of the relationships between trait-guilt and OC symptoms, the original proposal that appraisals of responsibility for harm evoke guilt and depression, which may worsen OC symptoms, seemed to be supported by earlier studies, strongly suggesting that the association between guilt and OCD is specific to patients with obsession about responsibility for causing harm or making mistakes and checking rituals. ${ }^{34-36}$ However, previous observations were mixed, and a more recent study ${ }^{37}$ found that trait-guilt did not predict any OC symptoms while disgust propensity was predictive of the contamination and symmetry OC symptoms. Overall, previous studies that have examined the relationships between cognitive-behavioral constructs such as between trait-guilt and OC symptoms have inconsistent findings. These mixed findings may stem from the limitations of the OCI-R itself, in which only symptoms that match the specific items on each subscale can be measured. Another problem is that each subscale contains only three items.

This study has some limitations. First, hierarchical regression analyses in this study showed that the inclusion of the three TAF subscales explained less than $10 \%$ of the additional variance, although this inclusion was significant in certain domains or symptoms. Second, all data were self-reported and several subscales with small item numbers such as TAF-LO and TAF-LS deviated from normality. Although parametric tests are acceptable because distribution of the main variables was normal and they were also used in previous studies, interpretation of the results should be done cautiously. Third, the generalizability of our results may be limited by the relatively small sample size along with the subjects in this study being almost all male. However, previous literature has suggested that TAF belief may be independent of gender. ${ }^{38,39}$ On the contrary, the fact that the subjects were relatively young with a narrow age range lessened the heterogeneity of our sample. Lastly, the potential effects of medication on dysfunctional beliefs 
are worth mentioning because over half of the patients were taking medications in this study. One study on patients with OCD showed significant changes in obsessive beliefs after 12 weeks of SSRI treatment, suggesting that the alleviation of negative mood by SSRIs reduces their dysfunctional appraisals. ${ }^{40}$ Therefore, our psychological data possibly included the medication effects given the cross-sectional nature of our study. Overall, our observations were preliminary in nature due to aforementioned limitations and further research with larger sample sizes is needed to delineate more clearly the character of the association between thought-action fusion and $\mathrm{OC}$ symptom dimensions.

In conclusion, these findings showed that TAF was closely related to two symptom domains, namely, the responsibility for harm and unacceptable thoughts domains. Inclusion of the three TAF subcomponents beyond inflated responsibility and trait-guilt explained the additional but small amount of variance in these two domains. In particular, TAF-LO and TAFLS were significant predictors for the unacceptable thoughts domain in opposite directions. In contrast, these findings were largely not reached in the same regression using OCI-R. Overall, this study suggests that the three TAF subcomponents may predict certain OC symptom dimensions, and a dimensional approach may complement typical symptom-oriented OC measures.

\section{Acknowledgments}

This work was supported by Biomedical Research Institute grant, Kyungpook National University Hospital (2016).

\section{Conflicts of Interest}

The authors have no potential conflicts of interest to disclose.

\section{Author Contributions}

Conceptualization: Seung Jae Lee, Ji Eun Kim. Data curation: Seung Jae Lee, Ji Eun Kim. Formal analysis: Seung Jae Lee, Ji Eun Kim. Funding acquisition: Seung Jae Lee. Investigation: Seung Jae Lee. Methodology: Seung Jae Lee, Ji Eun Kim. Project administration: Seung Jae Lee. Resources: Seung Jae Lee. Software: Seung Jae Lee, Ji Eun Kim. Supervision: Seung Jae Lee. Validation: Seung Jae Lee, Ji Eun Kim. Visualization: Ji Eun Kim. Writing_original draft: Seung Jae Lee, Ji Eun Kim. Writing_review \& editing: Seung Jae Lee, Ji Eun Kim.

\section{ORCID iDs}

\section{Ji Eun Kim}

Seung Jae Lee

https://orcid.org/0000-0002-3227-0107 https://orcid.org/0000-0003-3648-9824

\section{REFERENCES}

1. American Psychiatric Association. Diagnostic and Statistical Manual of Mental Disorders (5th Ed). Arlington: American Psychiatric Association; 2013.

2. Clark DA. Cognitive-Behavioral Therapy for OCD. New York: The Guilford Press; 2004.

3. Frost R, Steketee G. Cognitive Approaches to Obsessions and Compulsions: Theory, Assessment, and Treatment. Boston: Elsevier; 2002.

4. Salkovskis PM. Obsessional-compulsive problems: a cognitive-behav- ioural analysis. Behav Res Ther 1985;23:571-583.

5. Leckman JF, Bloch MH, King RA. Symptom dimensions and subtypes of obsessive-compulsive disorder: a developmental perspective. Dialogues Clin Neurosci 2009;11:21-33.

6. Abramowitz JS, Deacon BJ, Olatunji BO, Wheaton MG, Berman NC, Losardo D, et al. Assessment of obsessive-compulsive symptom dimensions: development and evaluation of the Dimensional Obsessive-Compulsive Scale. Psychol Assess 2010;22:180-198.

7. Julien D, O'Connor KP, Aardema F, Todorov C. The specificity of belief domains in obsessive-compulsive symptom subtypes. Pers Individ Differ 2006;41:1205-1216.

8. Tolin DF, Brady RE, Hannan S. Obsessional beliefs and symptoms of obsessive-compulsive disorder in a clinical sample. J Psychopathol Behav Assess 2008;30:31-42.

9. Myers SG, Fisher PL, Wells A. Belief domains of the Obsessive Beliefs Questionnaire-44 (OBQ-44) and their specific relationship with obsessive-compulsive symptoms. J Anxiety Disord 2008;22:475-484.

10. Tolin DF, Woods CM, Abramowitz JS. Relationship between obsessive beliefs and obsessive-compulsive symptoms. Cognit Ther Res 2003;27: 657-669.

11. Baer L. Factor analysis of symptom subtypes of obsessive compulsive disorder and their relation to personality and tic disorders. J Clin Psychiatry 1994;55(Suppl):18-23.

12. Leckman JF, Grice DE, Boardman J, Zhang H, Vitale A, Bondi C, et al. Symptoms of obsessive-compulsive disorder. Am J Psychiatry 1997;154: 911-917.

13. Rosario-Campos MC, Miguel EC, Quatrano S, Chacon P, Ferrao Y, Findley D, et al. The Dimensional Yale-Brown Obsessive-Compulsive Scale (DY-BOCS): an instrument for assessing obsessive-compulsive symptom dimensions. Mol Psychiatry 2006;11:495-504.

14. Deacon BJ, Abramowitz JS. The Yale-Brown Obsessive Compulsive Scale: factor analysis, construct validity, and suggestions for refinement. J Anxiety Disord 2005; 19:573-585.

15. Wheaton MG, Abramowitz JS, Berman NC, Riemann BC, Hale LR. The relationship between obsessive beliefs and symptom dimensions in obsessive-compulsive disorder. Behav Res Ther 2010;48:949-954.

16. Viar MA, Bilsky SA, Armstrong T, Olatunji BO. Obsessive beliefs and dimensions of obsessive-compulsive disorder: an examination of specific associations. Cognit Ther Res 2011;35:108-117.

17. Reuman L, Buchholz J, Abramowiz JS. Obsessive beliefs, experiential avoidance, and cognitive fusion as predictors of obsessive-compulsive disorder symptom dimensions. J Contextual Behav Sci 2018;9:15-20.

18. Bailey BE, Wu KD, Valentiner DP, McGrath PB. Thought-action fusion: Structure and specificity to OCD. J Obsess-Complus Rel 2014;3:39-45.

19. Rachman S. Obsessions, responsibility and guilt. Behav Res Ther 1993; 31:149-154.

20. Shafran R, Thordarson DS, Rachman S. Thought-action fusion in obsessive compulsive disorder. J Anxiety Disord 1996;10:379-391.

21. Rachman S, Shafran R. Cognitive distortions: Thought-action fusion. Clin Psychol Psychother 1999;6:80-85.

22. Kim HW, Kang JI, Kim SJ, Jhung K, Kim EJ Kim SJ. A validation study of the Korean-version of the dimensional obsessive-compulsive scale. J Korean Neuropsychiatr Assoc 2013;52:130-142.

23. Foa EB, Huppert JD, Leiberg S, Langner R, Kichic R, Hajcak G, et al. The Obsessive-Compulsive Inventory: development and validation of a short version. Psychol Assess 2002;14:485-496.

24. Woo CW, Kwon SM, Lim YJ, Shin MS. The Obsessive-Compulsive Inventory-Revised (OCI-R): psychometric properties of the Korean version and the order, gender, and cultural effects. J Behav Ther Exp Psychiatry 2010;41:220-227.

25. Lee S. The Relationships of OC Symptoms with Moral and Causal Responsibility and with Omission. Seoul: Department of psychology, Seoul National University; 2000.

26. Jones WH, Schratter AK, Kugler K. The Guilt Inventory. Psychol Rep 2000;87:1039-1042. 
27. Beck AT, Ward CH, Mendelson M, Mock J, Erbaugh J. An inventory for measuring depression. Arch Gen Psychiatry 1961;4:561-571.

28. Lee YH, Song JY. A study of the reliability and the validity of the BDI, SDS, and MMPI-D scales. Kor J Clin Psychol 1991;10:98-113.

29. Berle D, Starcevic V. Thought-action fusion: review of the literature and future directions. Clin Psychol Rev 2005;25:263-284.

30. Abramowitz JS, Whiteside S, Lynam D, Kalsy S. Is thought-action fusion specific to obsessive-compulsive disorder?: a mediating role of negative affect. Behav Res Ther 2003;41:1069-1079.

31. Reuman L, Buchholz J, Blakey SM, Abramowitz JS. Uncertain and fused: cognitive fusion and thought-action fusion in the context of obsessivecompulsive symptom dimensions. J Cogn Psychother 2017;31:191-203.

32. Shafran R, Rachman S. Thought-action fusion: a review. J Behav Ther Exp Psychiatry 2004;35:87-107.

33. Abramowitz JS, Lackey GR, Wheaton MG. Obsessive-compulsive symptoms: the contribution of obsessional beliefs and experiential avoidance. J Anxiety Disord 2009;23:160-166.

34. Foa EB, Amir N, Bogert KV, Molnar C, Przeworski A. Inflated perception of responsibility for harm in obsessive-compulsive disorder. J Anxiety Disord 2001;15:259-275.
35. Foa EB, Sacks MB, Tolin DF, Prezworski A, Amir N. Inflated perception of responsibility for harm in OCD patients with and without checking compulsions: a replication and extension. J Anxiety Disord 2002;16: 443-453.

36. Salkovskis PM, Wroe AL, Gledhill A, Morrison N, Forrester E, Richards $\mathrm{C}$, et al. Responsibility attitudes and interpretations are characteristic of obsessive compulsive disorder. Behav Res Ther 2000;38:347-372.

37. D’Olimpio F, Cosentino T, Basile B Tenore K. Obsessive-compulsive disorder and propensity to guilt feelings and to disgust. Clin Neuropsychiatry 2013;10:20-29.

38. Muris P, Meesters C, Rassin E, Merckelbach H, Campbell J. Thoughtaction fusion and anxiety disorders symptoms in normal adolescents. Behav Res Ther 2001;39:843-852.

39. Yorulmaz O, Yilmaz AE Gencoz T. Psychometric properties of the Thought-Action Fusion Scale in a Turkish sample. Behav Res Ther 2004;42:1203-1214.

40. Selvi Y, Atli A, Besiroglu L, Aydin A, Gulec M. The impact of obsessive beliefs on pharmacological treatment response in patients with obsessive-compulsive disorder. Int J Psychiatry Clin Pract 2011;15:209-213. 